\title{
Design and Development of in-home Wireless Crucial Events Logging and Alarming System for Elderly and Disabled People Care
}

\author{
Ali S. Al-Mejrad \\ Department of Clinical Laboratory Sciences, Faculty of Applied Medical Sciences University of Hail, Hail, \\ Kingdom of Saudi Arabia. \\ E-Mail:a.almejrad@uoh.edu.sa
}

\begin{abstract}
This paper aims at developing a system that will help in in-home care for elderly or disabled people. The system can record the patient vital parameters such as temperature, $\mathrm{SpO} 2, \mathrm{ECG}$, heart rate, bed moisture and fall off sensor which could be installed at the bathroom or shower area at the patient terminal and transmit the critical situations such as high or low values to an alarming system. The system will be developed using the National Instrument Wireless Sensor Network (NI-WSN) to control the crucial events transmission and LabVIEW software to design the user interface.
\end{abstract}

Keywords: Elderly; Disabled; In-home care; NI-WSN; PSoC; LabVIEW.

\section{Introduction}

The growth in the number and proportion of elderly and disable people are significantly increase which lead to the need of a special care system to give these people a chance to live depending on themselves [1]. In addition, the resources in in-patient facilities are limited and expensive. Elderly people are suffering many health problems, mobility limitation, and chronic physical problems and, other disorders compared to younger age people. All of which are requiring special continuous attention and care. The family and/or other help provider often shares the task of caring for these people. However, the elderly usually remain independent and alone at home and this becomes a critical issue due to non-availability of their help provider. While at home, smart sensors such as body temperature and ECG sensors can be used to wirelessly connect through NI-WSN to a Computer, which will record and analyze different parameters such as, body temperature, blood pressure, cardiac function and many others [2]-[4]. A connection to the Internet keeps the Elderly and disabled people within reach of their help providers and family. This method of transmitting selected data only when required rather than streaming all data continuously, is perfect method for monitoring critical situations and activating alarms for these critical cases [5]-[8]. Elderly people in Saudi
Arabia are suffering from chronic health conditions such as chronic cardio-respiratory diseases, which is one of the major causes of death and admission to hospital in addition to many other concurrent problems [9]. Due to frequent occurrences of such diseases to these people who in need for continuous care will lead to costly hospitalization of such patients. In this paper a system will be developed that will help in in-home care for elderly or disabled people by recording the patient vital parameters such as SPO2, heart rate, ECG, temperature, bed moisture, and fall off sensor on the chair or the bed. In addition, these sensors could be installed at the bathroom or shower area at the patient terminal and transmit the critical situations such as high or low values to an alarming system. The system will be developed using the National Instrument Wireless Sensor Network (NI-WSN) to control the curial events transmission and LabVIEW software to design the user interface.

\section{System Development}

The designed system in the block diagram consists of different components as shown in Figure. 


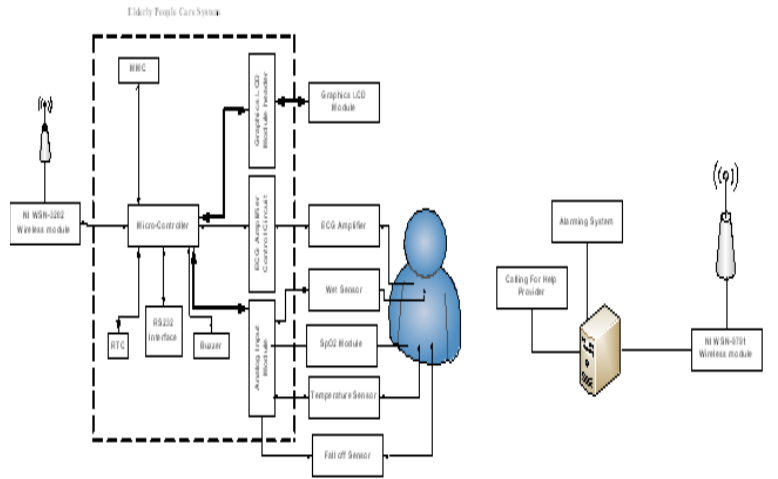

Figure 1. Block Diagram of the Designed System

The system has two major components; the console that is fixed at the patient chair, bed, or even held with the patient with the NI-WSN-3202 wireless module [10] and the other component is the NI-WSN-9791 Ethernet gateway [11] connected to the PC that is also connected to an alarming system to trigger alarm on critical situations. A user interface program using LabVIEW is developed to control the wireless module and collect the data of patient events such as high heart rate, high temperature, and fall off the chair or bed [12].

\section{Measurement node NI-WSN-3202:}

The measurement nodes connect directly with sensor and are able to transmit wirelessly a $2.4 \mathrm{GHz}$ radio data to the WSN Ethernet gateway. Each measurement node has four analog input channels and four digital Input/output channels.

The measurement nodes and the NI WSN-9791 Ethernet gateway:

The measurement nodes and the Ethernet gateway communicate wirelessly using a radio frequency of 2.4 $\mathrm{GHz}$ based on the reliable IEEE 802.15.4 NI WSN protocol. The network can accommodates up to 36 nodes per gateway and provide an outdoor range of up to $300 \mathrm{~m}$.

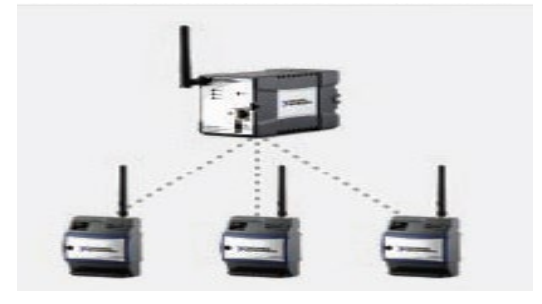

Figure 2. NI Wireless Sensor Network

\section{Microcontroller PSoC (CY8C29466-24PVXI)}

Programmable System on Chip (PSoC) is a programmable embedded system on chip which integrating on a single chip configurable digital and analog peripheral functions, memory and a microcontroller [13]. PSoC is designed to replace components in traditional MCU-based system with a single-chip programmable device. PSoC device has configurable blocks of digital and analog, as well as programmable interconnections. This architecture enables designer for creating customized peripheral configurations. This microcontroller is responsible for data collecting from all the analog modules connected to the patient and preparing the data to be transferred to/from the wireless module. Also to keep a complete trend in the MMC module as a data logger.

\section{Results and Discussion}

First, a prototype test system of three main signals including pulse oximeter, temperature and ECG using PSoC is developed to ensure the success of the data collection. The block diagram of the prototype test system with LCD shown in Figure 3 consisting of three main traces are described below: 


\section{ADC Converter}

The analog to digital converter used to acquire the signal in digital form in order to display it on the LCD; here we use the PSoC 12 bits ADC converter with sampling rate of 50 .

Now the signal will be handled by to the PSoC internal controller in order to display it on the LCD.

\subsection{Temperature}

\section{$P G A$}

Figure 3. Prototype test system of three main signals including pulse oximeter, temperature and ECG using PSoC

\subsection{Pulse Oximeter:}

First module is Pulse Oximeter, the frequency of this signal in the range of $1 \mathrm{~Hz}$ to $40 \mathrm{~Hz}$ the sensor used in this module is photo sensor sender and receiver, the finger of the patient located between the them, the infrared opt coupler will detect the volume change in finger's capillaries and to obtain blood flow profile.

\section{Programmable Gain Amplifier (PGA)}

The received light is converted into an electric signal by a photodiode and then enters the first block inside the PSoC controlled is PGA here which act as a buffer to match the circuit input output impedance, and also amplify with gain of 4 .

\section{Filters}

The second block is filter we use two types of filter the first is second order Low pass filter, this filter is used to cut the high frequency component, the corner frequency of the filter is $100 \mathrm{~Hz}$, the other filter is Band pass filter is used to cut plethysmograph signal band $(1-40 \mathrm{~Hz})$

\section{Pulse Peak detector and pulse counter}

The function of this block is to detect the peaks of the signal and pass it to the pulse counter, which is a digital counter in order to count the heart rate.
The PGA here amplifies the sensing signal from the bridge circuit and pass it to the ADC converter.

\section{ADC Converter}

Converts the thermistor voltage into digital form to the PSoC internal controller to check the calibration tables and display the temperature on the LCD by using the graphic LCD routine.

\section{$3.3 E C G$}

\section{Instrumentation Amplifier}

In our system, some external circuits (outside the PSoC controller) were made such as Instrumentation amplifier and isolator circuit because of some limitation in the ranges of the PSoC.

\section{Filters}

The second block is filter. Two types of filter were used the first is second order Band Pass filter, this filter is used to cut the high and low noise and pass only the ECG signal $(1-150 \mathrm{~Hz})$, the other filter is Notch filter is used to cut mains noise $60 \mathrm{~Hz}$. The response of the amplifier circuit has been verified using Orcad Pspice simulation program and the response is illustrated in Figure 4 bellow

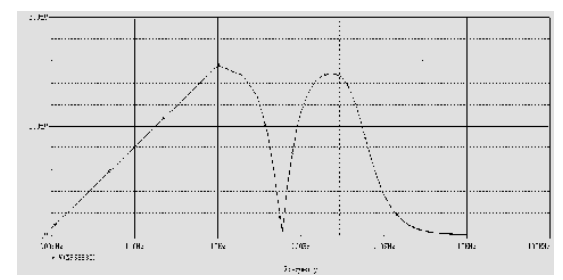


Figure 4. The total ECG filter response $P G A$

Act here as the main amplifier of the ECG signal, and uses a gain of 16 .

\section{ADC Converter}

The analog to digital converter used to acquire the signal in digital form in order to display it on the LCD; here we use the PSoC 12 bits ADC converter with sampling rate of 50 .

Now the signal will be handled by to the PSoC internal controller in order to display it on the LCD.

\subsection{Designed PSoC Circuit Diagram}

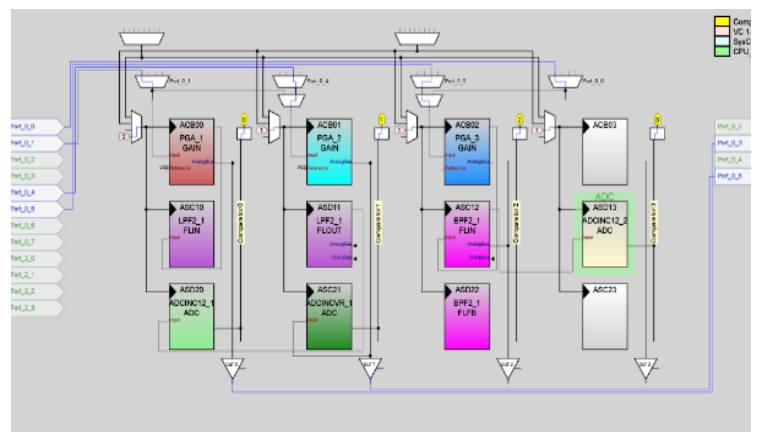

Figure 5. Designed PSoC circuit diagram

The analog signals of the three signals sampled in PSoC micro-controller designed as shown in Figure 5 are then stored in the system EEPROM and/or the MMC card on the system shown in Figure 1 for later analysis. The digital data is wired to the measurement node NI-WSN-3202 that is responsible for the wireless transfer of the data to the NI WSN-9791 Ethernet gateway receiver. The receiver node is connected to the PC through the Ethernet port for data collection and alarm triggering. Experimentation utilizing the prototype based on wireless technology with the PSoC system has shown that the communication range varies from 30 to 50 meters depends on the home walls that is sufficient.
The complete system components is shown in Figure 6 and hardware test setup is shown in Figure 7.

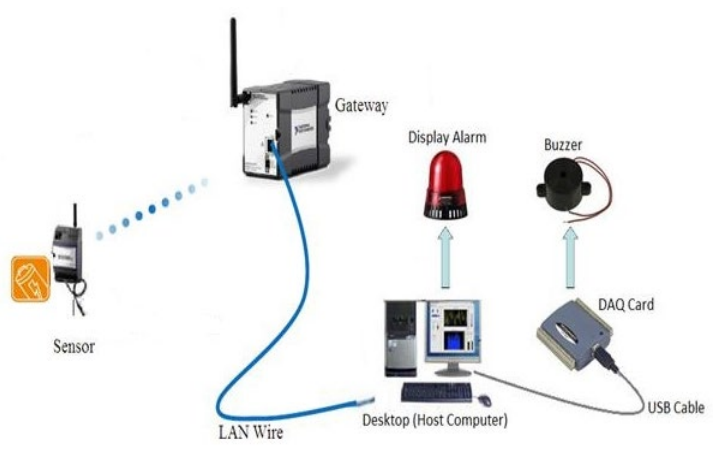

Figure 6 . The complete system components

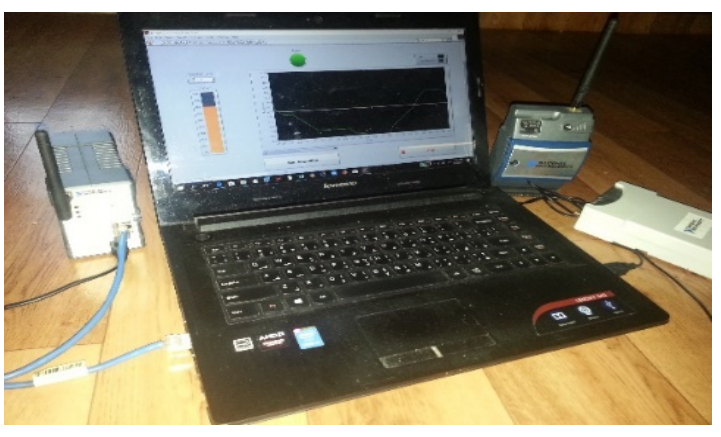

Figure 7. Hardware test setup with user interface

The system includes main components including a user interface program as shown in Figure 8. The system is developed to control the whole system by logging in the system and then configuring the events with minimum and maximum limits and configuring wireless module. When running, the system starts patient data acquisition, and display of the data and events of the patient such as high temperature, high heart rate, low oxygen saturation, and fall off the chair or bed. Finally, patient's data and events can be analyzed for follow-up and diagnosis purposes.

\subsection{Designed System and its User Interface}




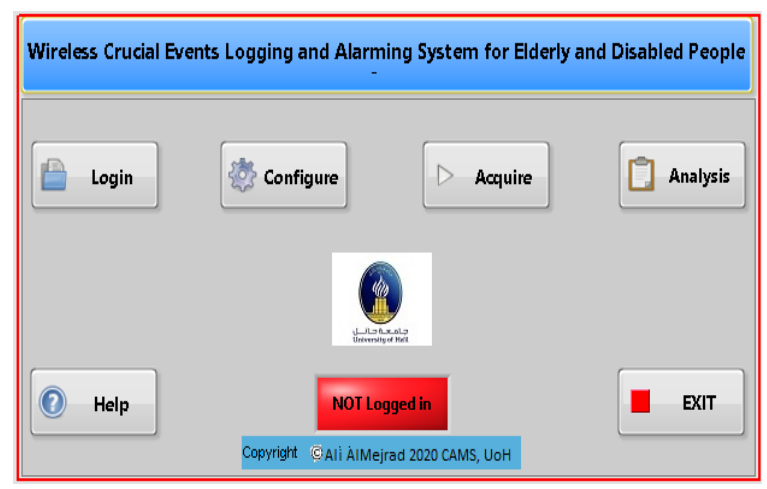

Figure 8. User Interface System

\section{Conclusion and Future Work}

The designed system goal is to provide in home, selfdependent, monitoring for critical events, and alarming system. As the majority of elderly people prefer to stay in their own homes and may require help and supervision. The designed system is monitoring the vital signs of the patient such as $\mathrm{SpO} 2$, heart rate, temperature, ECG, and the critical status (wet clothes and fallen off the chair) which are added easily as $\mathrm{ON} / \mathrm{OFF}$ in order to trigger alarms for such events.

The system is connected to a PC that can easily transfer the collected data on the web for long distance further data analysis and/or call for help. This continuous remote monitoring and support will increase the elderly people freedom and safety; and prevent patient condition deterioration. This in turn will improve the elderly life quality. The designed system was developed as a prototype test system including three signals including pulse oximeter, temperature and ECG using PSoC with LCD. The future work is all about reducing the component using the Analog Front End (AFE) chips to improve power consumption and reduce the cost.

\section{Acknowledgment}

I like to thank the Deanship of Scientific Research of University of Hail, for their support of my research.

\section{References}

1- United Nations, Department of Economic and Social Affairs, Population Division. World Population Ageing 2015 (ST/ESA/SER.A/390), 2015.

2- Eduardo Freitas and Amandio Azevedo, "Wireless Biomedical Sensor Networks: The Technology", Proceedings of the 2nd World Congress on Electrical Engineering and Computer Systems and Science (EECSS'16) Budapest, Hungary - August 16-17, 2016.

3- Panicker N. and A. Sukeshkumar, "Wireless sensor based systems for biomedical monitoring: A review", AMSE JOURNALS -2014-Series: Modelling C; Vol. 75; No. 1; pp 65-78, 2014.

4- Demongeot, J. et al.,"Multi-sensors acquisition, data fusion, knowledge mining and alarm triggering in health smart homes for elderly people", C. R. Biologies 325:673-682, 2002.

5- Clark, A. and K. EMMA, "A comprehensive alarm system for the elderly and infirm and disabled who live at home", Res. Soc. Health Care, 15:205-27, 1979.

6- Chan, M. et al.,"Smart house automation system for the elderly and the disabled", IEEE Int. Conf. Syst. Man Cybern, 2:1586-1589, 1995

7- Peeters, P. H., "Design criteria for an automatic safetyalarm system for elderly", Technol. Health Care, 8:8191,2000.

8- A. Tzavaras, and B. Spyropoulos, “ Development of a low-cost wireless monitoring System supporting the Continuity of Medical Care of the Patient at home", 35th Annual International Conference of the IEEE EMBS Osaka, Japan, 3 - 7 July, 2013

9- Khan J. et al., "The Saudi Guidelines for the Diagnosis and Management of COPD", Ann Thorac Med. 9(2): pp. 55-76, 2014.

10- NI-WSN-3202 wireless module - User guide and Specifications, 2010.

11- NI WSN-9791 Ethernet Gateway - User guide and Specifications, 2010.

12- National Instruments Corporation, "LabVIEW User Manual", Edition 2014.

13- Cypress Semiconductors, PSoC 5LP Architecture Technical Reference Manual, Document No. 00178426 Rev. *F, 2016. 